\title{
The Development of a Methodology for Gamifying Surveys
}

\author{
Casper Harteveld \\ Northeastern University \\ c.harteveld@northeastern.edu \\ Jack Hart \\ Northeastern University \\ hart.jo@husky.neu.edu

$\begin{array}{ll}\text { Sam Snodgrass } & \text { Tyler Corwin } \\ \text { Northeastern University } & \text { Northeastern University } \\ \text { s.snodgrass@northeastern.edu } & \text { corwin.t@husky.neu.edu }\end{array}$

Omid Mohaddesi

Guillermo Romera Rodriguez

Northeastern University

Northeastern University

mohaddesi.s@northeastern.edu romerarodriguez.g@husky.neu.edu

Permission to make digital or hard copies of all or part of this work for personal or classroom use is granted without fee provided that copies are not made or distributed for profit or commercial advantage and that copies bear this notice and the full citation on the first page. Copyrights for components of his work owned by others than ACM must be honod. Abstractig wh credit is permitted. To copy otherwise, or republish to post on servers or to redistribute to lists, requires prior specific permission and/or Ce. RequY

ne, Australia.

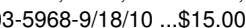

https://doi.org/10.1145/3270316.3271544

\begin{abstract}
While some promising early work exists, gamified surveys are still not widespread. In this work-in-progress paper, we present our methodology on gamifying surveys, which combines the rigor of survey design with the creativity and affordances of game design and makes use of an existing tool to ease development. We illustrate our methodology with four different gamified surveys focused on personality tests and frameworks-in general and in the context of games. We discuss the challenges and opportunities and describe a research agenda to advance this work.
\end{abstract}

\section{CCS Concepts}

-General and reference $\rightarrow$ Measurement; $\cdot$ Human-centered computing $\rightarrow \mathrm{HCl}$ design and evaluation methods;

\section{Author Keywords}

Gamification; gamified surveys; games as research instruments; methodology; survey design.

\section{Introduction}

Currently, gamifying surveys is not a widespread practice [11]. Early experimental examples exist, one where, for example, participants control a spaceship and have to shoot the answers they want to choose [12]. Such examples immediately highlight one of the issues with gamifying surveys, which is the trade-off between user experience 


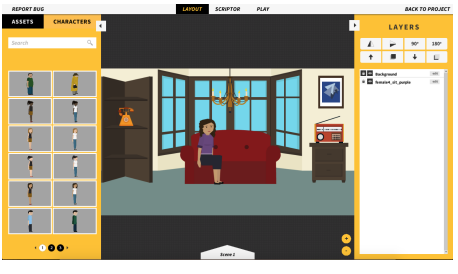

Figure 1: Layout tool to visualize settings. Users can choose backgrounds, objects, characters, and interface elements from a list and place them on a canvas c) Northeastern University.

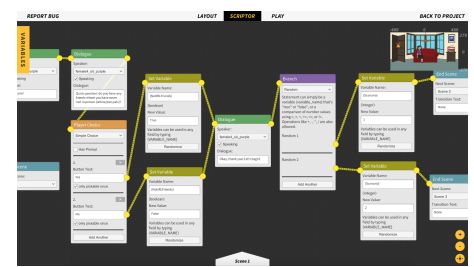

Figure 2: Script tool to make a visual decision tree with nodes and links for how a scenario unfolds and to specify the dialog and actions (C) Northeastern University.

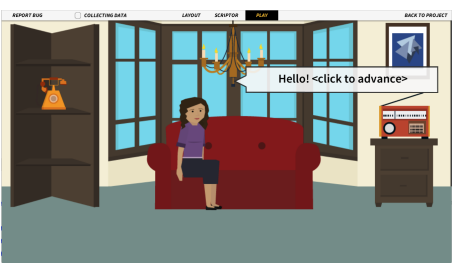

Figure 3: Play tool to playtest projects $\odot$ Northeastern University. and usability. Other efforts are less radically different in interaction, relying more on instrumental (points, progress bars, badges) or aesthetic (sounds, animations, graphics) aspects. The consideration to gamify surveys is based on a need to better engage survey participants. While surveys have various methodological advantages, such as that they are relatively easy to develop, deploy, and analyze and are capable of collecting data from a large number of respondents, they suffer in particular from a lack of engagement, resulting in a low response rate, speeding, random responding, premature termination, and a lack of attention $[7,8]$. It is conjectured that through the use of game design participation will increase and yield higher quality data [2]. Results thus far are promising, showing positive effects in, among others, attitude and time spent without introducing strong biases [1].

One important reason why gamifying surveys may not be taking off is that they nullify the advantages of surveys.

Turning a survey into an interactive (game) experience is hard: it requires significant time and resources and also skills, creativity, and expertise in thinking how an otherwise traditional survey can be transformed. There are also concerns about the validity of responses and how the type of experience may attract or deter audiences, and thus cause a selection bias [1]. Aside from empirical evidence, what is currently especially lacking to advance this area are both methodologies and tools that will help to create gamified surveys. There is no "SurveyMonkey" or "Qualtrics" for gamified surveys and no established practices such as using Likert or semantic differential scales.

To contribute to this emerging area, in this work-in-progress paper we present our methodology to developing gamified surveys. We developed four different gamified surveys, each based on either a theoretical framework or traditional survey, using a new easy-to-use platform called Study Crafter [9]. We first discuss recent efforts in this area. We then introduce our design methodology. Then, we detail each of the surveys, focusing on their similarities and differences. We end with a discussion of the challenges and opportunities and a research agenda to advance this work.

\section{Related Work}

There have been several classifications of how to gamify surveys to help distinguish the different approaches. At its most basic, Bailey et al. [2] and Ewing [4] describe two categories that are useful: soft gamification, the addition of minor game elements to a traditional survey (e.g., badges, more visual aspects, different interaction methods); and hard gamification, the complete redesign of a traditional survey into a game-like experience. Downes-le Guin et al. [7] propose four categories that provide more granularity on soft gamification: Text Only is a traditional survey; Decoratively Visual includes visual elements to enhance user engagement; Functionally Visual includes enhancements to the choice interactions to increase engagement (e.g., sliders or different choice mechanics); and Gamified includes any addition of game elements ranging from simple additions (e.g., badges, progress indicators) to full game experiences. For our work, we focused on full game experiences or "hard gamification" because it leverages the affordances of games the most, fostering intrinsic motivation and collecting data in a controlled but unobtrusive manner [10].

In terms of helping to design gamified surveys, to our knowledge there are only two substantive attempts on developing a methodology for gamified surveys: Harms et al. [8] describe how to integrate the MDA framework with survey design and Puleston [12] outlines a number of techniques to reframe questions and provides a number of suggestions of how to design questions to be more game-like. The lack is 


\section{Big 5 Personality Traits}

Openness to Experience:

The extent to which a person appreciates adventure and creativity, and is able to think about abstract concepts.

Conscientiousness: The extent to which a person is thoughtful, organized, and detail-oriented.

Extraversion: Extent to which a person is social emotionally expressive, and assertive.

Agreeableness: Extent to which a person is trusting, empathetic, and altruistic.

Neuroticism: The extent to which a person is prone to stress, irritability, and sadness.

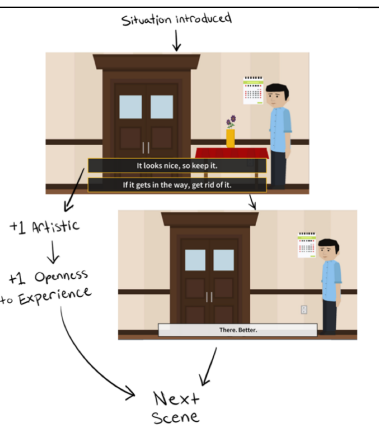

Figure 4: Scoring Big Five. (C) Northeastern University. not surprising. In 2017, a literature review from Keush and Zhang [11] found that despite the wide interest in gamified surveys only 14 studies have been conducted on the topic at large. We believe that easy-to-use tools to create gamified surveys will help advance this area and, therefore, in our work we coupled the use of such a tool with thinking on how to develop gamified surveys. For this development, we focused in our methodology more on recreating the rigor in survey design as opposed to primarily considering the creative process of making it more game-like.

\section{Design Methodology}

A typical survey consists of a set of closed questions supported by a number of open questions. The closed questions require respondents to choose among a predefined set of options and aim to capture their beliefs, perceptions, and attitudes on particular topics and/or reveal latent constructs that may explain the choices they made, such as personality traits or underlying motivations. In our methodology, we take these measures as the starting point. In fact, the very first step is to identify the concepts that are to be measured. If gamifying an existing framework or theory, then this corresponds to the facets of that framework. Taking the Big Five Personality traits model as an example, the concepts to be measured could be the five personality traits, or they could be the supporting facets of each of the traits, or some combination thereof.

Next, we decide how to gamify those concepts. This step can be performed in a variety of ways, and in fact, in our example surveys presented in this paper we experiment with several such approaches. One approach, if using a model with an existing traditional survey, is to leverage the existing survey questions and directly encode them into gamelike experiences. For example, a question about whether the participant enjoys exploration can be encoded into a game environment where the participant has the option to explore. We use this direct encoding approach when gamifying the Self Determination Theory model and the Player Types model. Another approach is to create game-like experiences that are meant to measure a participant's score in the chosen concepts. This approach requires more design, as measuring abstract concepts can be more difficult to encode than concrete questions. For example, if one of the concepts being measured is the participant's extraversion (as in the Big 5), then we can create a game-like experience where the participant has the opportunity to interact with a variety of game characters. However, in these scenarios we must be careful to not introduce biases into the scenarios to ensure that we are actually measuring extraversion and not simply a preference for talking to specific people or characters.

Lastly, we need to decide how the scoring of such interactions can be done in a rigorous manner. Many traditional surveys use a Likert scale and use repeated questions to measure the same concept. To ensure the same level of granularity and validity, it is important to have multiple scenarios for a given concept in a gamified survey.

To create our gamified surveys we used StudyCrafter [9], a platform developed for professional researchers to conduct the next generation of research, fostered by game-based and crowdsourced technologies. With StudyCrafter users can create gamified projects with a simple patch programming and scene layout interface. Figures 1-3 show the main StudyCrafter project creation tools.

\section{Gamified Surveys}

To illustrate the use of our methodology, we developed gamified versions of several personality tests and frameworksin general and in the context of games. In this section we 


\section{SDT Facets}

Autonomy: This corresponds to a person's need to feel as though they have control over their life.

Competence: This corresponds to a person's need to build skills and develop mastery over tasks.

Relatedness: This corresponds to a person's need to have a sense of belonging and connectedness.

\section{Play Facets}

Assault: Interested in violence, destruction, and aggression.

Manage: Interested in building, reaching agreements, and commanding groups.

Journey: Interested in exploration of the game world and back story of the character they were playing.

Care: Interested in romance, taking care of pets, and other similar emotional actions.

Coordinate: interested in platformers, rhythm games, and other skill or puzzlebased games describe how we represent the original chosen surveys and frameworks as gamified interactive experiences.

\section{Big Five Personality Traits}

The Big Five Personality Traits (Big 5) [6] is a model for describing personality based on the association between words to describe individuals with similar aspects of personality. For example, someone who is referred to as "extraverted" is likely to be called "outgoing" and not "shy." The theory proposes five dimensions: openness to experience, conscientiousness, extraversion, agreeableness, and neuroticism (see sidebar). Additionally, each dimension is measured along six facets that individuals can be stronger or weaker in. For example, Conscientiousness has facets corresponding to orderly vs. disorganized and impulsive vs. cautious, whereas Agreeableness has facets corresponding to skeptical vs. trusting and modest vs. conceited.

We built a narrative-based game based on these facets as a way to create a profile for the participant (Fig. 5). At a high level, we crafted specific interactions and choices to measure a participant's penchant for each facet of each trait. Notice, we only create a single choice or interaction for each facet in order to maintain a reasonable length for the gamified survey. The gamified survey takes place during a business week with the participant playing the role of a businessman. The participant is put into situations as the businessman and must make choices based on how they would act. For example, in one scene the participant is shown a decorative table and told that they frequently hurt themselves by walking into that table. They are then asked if they would rather keep the table or remove it to prevent further injury. In this scenario, the choice to keep or remove the decorative table evaluates the participant on the Artistic vs. Practical facet of the Openness to Experience trait. Once the participant completes every scenario, they are shown how they scored in each trait, along with whether or not they scored in each facet of that dimension. For example, if the participant chose to keep the table, then they scored towards the Artistic facet which contributed to their overall Openness to Experiences score (Fig. 4).

\section{Self-Determination Theory}

Self-Determination Theory (SDT) [3] is a theory of motivation meant to explain a person's intrinsic motivations to behave in specific ways by measuring three facets: Autonomy, Competence, and Relatedness (see sidebar). Each facet relates to the different aspects of motivation (e.g., for Competence a person may be motivated to succeed or excel in certain activities, and for Autonomy a person may be motivated by having control over their actions and choices).

We built a narrative-based game meant to measure a participant's intrinsic motivations as described by the SDT framework (Fig. 6). Participants play as Michael, a student who has to go through the first day of school for a new year. At a high level, we constructed a series of scenarios that present choices to the participant; these scenarios and choices map directly to questions from the original SDT survey [5]. We constructed five such scenarios for each factor of the SDT framework. In one scene the participant must choose several classes for Michael. The participant is given the option between a class that Michael's friends are taking and a class that Michael is more interested in. Choosing to enroll in the class that Michael is more interested in awards the player with points toward their Autonomy score, whereas choosing the other option awards no points. This specific scenario is a gamification of the question "I feel like I am free to decide for myself how to live my life."

Player Types

The player types model developed by Vahlo et al. [14] used a data-driven approach to identify player types. In the study, 


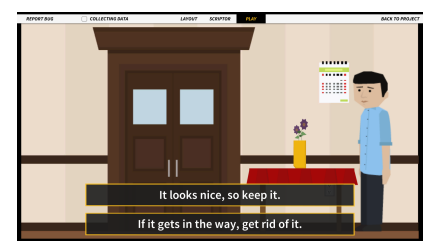

Figure 5: Big 5 @ Northeastern University.

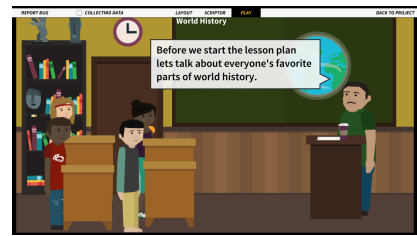

Figure 6: SDT @ Northeastern University.

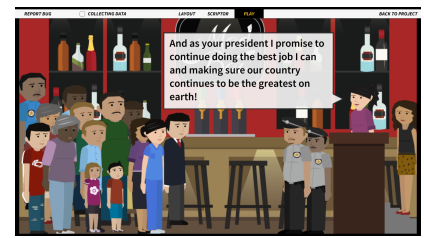

Figure 7: Player Types (c) Northeastern University.

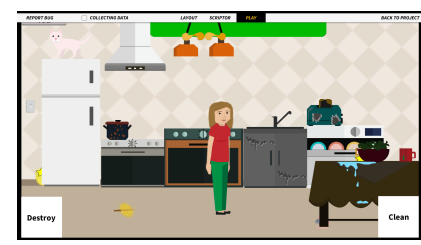

Figure 8: Motivation Model (c) Northeastern University. participants were asked to answer a questionnaire on their video game playing habits and preferences. Through their responses, five facets were identified (see sidebar). Analysis was then conducted to identify seven player types: Mercenary, Adventurer, Commander, Daredevil, Companion, Patterner, and Explorer. Each of these player types has unique characteristics in terms of the facets. For example, the Mercenary player type scores high in the Assault facet, but low in the Care facet.

We built a narrative-based game to measure the five facets and by extension identify the participant's player type (Fig. 7). Participants play the role of a woman running for reelection as the President of an unnamed country. As they are running for reelection, important decisions must be made to run a successful campaign. At a high level, for this gamified survey we follow a similar procedure to that of the Big 5 and SDT surveys. However, in addition to constructing scenarios that correspond to the different facets, we constructed mini-games to measure specific facets that are not easily captured by narrative scenarios. For example, to measure the Manage facet the participant was asked to create a budget for the campaign and plan a rally (or have the assistant manage both), but to measure the Coordinate facet the participant must play a Whack a Mole-style game of clicking objects as they appear. These choices are then analyzed to give players one of the seven player preferences.

Motivation Model

The Motivation Model based on the Gamer Motivation Model by Quantic Foundry [15] aims to categorize participants based on their playstyle and data analytics. There are six different categories: Action, Social, Mastery, Achievement, Immersion, and Creativity (see sidebar).

We built an action-based game meant to measure the different categories of the Gamer Motivation Model (Fig. 8).
In this gamified survey we take a much different approach than that of the previous surveys. Namely, we encode measurements of the categories into actions and gameplay elements that the participant can perform as well as choose. Additionally, whereas the previous gamified surveys use choices and actions that measure each facet individually (i.e., either contribute/subtract from the facet or not), this gamified survey offers the choice of alternatives which measures multiple facets simultaneously (i.e., option 1 contributes to one facet, whereas option 2 contributes to a different facet). In a way, this implementation is more like a semantic differential scale, whereas the previous gamified surveys are more like a Likert scale.

In this gamified survey the participant takes the role of a housekeeper, and throughout the experience the participant must make a variety of choices about how different elements of the experience are presented (e.g., what their character looks like, GUI elements, etc.) as well as perform actions in game (e.g., clean up or destroy certain objects). The final scores provided to the participant are the individual percentage scores for each category. That is, how well the participant fits into each category (e.g., $75 \%$ into the Action category and $45 \%$ in the Social category, etc.).

\section{Discussion}

In the previous sections we described our methodology and how we used that to create gamified versions of four personality and player profiling models. As noted in previous sections, there are two broad categories of gamifying surveys: soft gamification and hard gamification [2]. Within these categories there are subcategories. For example, soft gamification can mean making a more interactive or playful interface or simply trying to reframe the questions such that they are more immersive for the participant. Additionally, there are several ways to approach hard gamification, 


\section{Motivation Model}

Action: The participant enjoys a destructive, fastpaced, and chaotic playstyle.

Social: The participant enjoys team building, and playing cooperatively and competitively.

Mastery: The participant enjoys challenges, thinking ahead, and high difficulty.

Achievement: The participant enjoys a completionist playstyle, being a high level, completing all tasks, and collecting all items in the game.

Immersion: The participant enjoys being immersed in the experience, being another person, and enjoys interesting story lines.

Creativity: The participant enjoys expressing themselves, exploring, and tinkering with everything within their reach. as we show in the previous section. For example, we show that you can directly convert the survey questions into interactive scenarios (as we did with SDT) or more broadly create scenarios to measure different aspects of the models (as we did with the Player Preference and Big 5 surveys) or even develop mappings between gameplay elements and survey aspects (as we did with the Motivation Model). What our work contributes are the high-level steps and considerations to "hard" gamify surveys: identifying the concepts to be measured, deciding how to translate the concepts in a game-like experience, and scoring the results.

It is important to point out that each of the efforts we explored have potential failings. For instance, mapping gameplay elements to specific personality facets may lead to biased results if a participant does not enjoy the specific gameplay style or if the gameplay lends itself more easily to different player types. Additionally, placing a participant within a narrative context may bias them to behave how they believe the character would behave, and not necessarily how they themselves would behave. Lastly, a potential pitfall all gamified surveys need account for, is that by providing these game contexts to the participants, we may also introduce implicit opportunity costs to the questions that do not exist in the standard surveys (i.e., answering a question a certain way may open additional/different content than answering a question another way, whereas answering one question in a survey does not impact the participant's access to the rest of the survey). These potential drawbacks and biases can be tested for and explored more deeply through application of our surveys.

Lastly, we want to highlight that there has not been much work previously in hard gamification of surveys. This is likely due to the amount of knowledge (both of game design and surveys) required to create a proper gamified sur- vey and the effort required to implement such a survey. By leveraging the affordances of StudyCrafter we were able to rapidly prototype, playtest, and iterate on our gamified surveys. StudyCrafter as a tool can allow researchers to more easily implement and experiment with hard gamification by lowering the effort barrier in terms of implementation.

\section{Conclusion}

We show in this work-in-progress paper a methodology for developing gamified surveys. For this methodology, we combine the rigor of survey design with the creativity and affordances of game design, resulting in interactive experiences that aim to capture concepts in a non-obtrusive and engaging manner. We further showcase varying ways in which gamified surveys can be designed, and how this development process can be eased with the existence of a tool such as StudyCrafter, which in the future will arguably help with the further adoption and use of gamified surveys.

Our future work will focus first on validating the gamified surveys by actually implementing them, and comparing the results with traditional or the original surveys. A delicate issue in such comparative evaluation is that there is no ground truth. Second, by continuing our efforts in designing and implementing gamified surveys we will help to solidify a more detailed and refined methodology for gamified surveys. Third and finally, we recognize that gamified surveys still require significant effort and expertise and we will explore how to empower users with design assistance and creativity support [13] to accomplish their goals.

\section{Acknowledgements}

We thank everyone who contributed to the design of StudyCrafter and playtested our surveys. We further acknowledge the grant support from Northeastern University, DARPA (D16AP0011), and NSF (IIS-1736185). 


\section{REFERENCES}

1. Maree Ackehurst and Rose-Anne Polvere. 2016. Increasing survey engagement through gamification and the use of mobile devices. (2016).

2. Pippa Bailey, Gareth Pritchard, and Hollie Kernohan 2015. Gamification in market research: Increasing enjoyment, participant engagement and richness of data, but what of data validity? International Journal of Market Research 57, 1 (2015), 17-28.

3. Edward L Deci and Richard M Ryan. 2002. Handbook of self-determination research. University of Rochester Press.

4. Tom Ewing. 2012. Four types of gamification that can be used in market research. (2012). http:

//blackbeardblog.tumblr.com/post/13452542524/

state-of-play-four-types-of-research-gamification visited on August 3rd, 2018.

5. Marylène Gagné. 2003. The role of autonomy support and autonomy orientation in prosocial behavior engagement. Motivation and emotion 27, 3 (2003) 199-223.

6. Lewis R Goldberg. 1992. The development of markers for the big five factor structure. Psychological assessment 4, 1 (1992), 26.

7. Theo Downes-Le Guin, Reg Baker, Joanne Mechling, and Erica Ruyle. 2012. Myths and realities of respondent engagement in online surveys. International Journal of Market Research 54, 5 (2012), 613-633.

8. Johannes Harms, Christoph Wimmer, Karin Kappel, and Thomas Grechenig. 2014. Gamification of online surveys: conceptual foundations and a design process based on the MDA framework. In Proceedings of the 8th Nordic Conference on Human-Computer Interaction. ACM, 565-568.

9. Casper Harteveld, Nolan Manning, Farah Abu-Arja, Rick Menasce, Dean Thurston, Gillian Smith, and Steven C Sutherland. 2017. Design of playful authoring tools for social and behavioral science. In Proceedings of the 22nd International Conference on Intelligent User Interfaces Companion. ACM, 157-160.

10. Casper Harteveld and Steven C Sutherland. 2017. Personalized gaming for motivating social and behavioral science participation. In Proceedings of the 2017 ACM Workshop on Theory-Informed User Modeling for Tailoring and Personalizing Interfaces. ACM, 31-38.

11. Florian Keusch and Chan Zhang. 2017. A review of issues in gamified surveys. Social Science Computer Review 35, 2 (2017), 147-166.

12. Jon Puleston. 2011. Online research-game on!: A look at how gaming techniques can transform your online research. In Proceedings of the 6th ASC (Association for Survey Computing) International Conference Shifting the Boundaries of Research. Citeseer, 20-50.

13. Ben Shneiderman. 2007. Creativity support tools: Accelerating discovery and innovation. Commun. ACM 50, 12 (2007), 20-32.

14. Jukka Vahlo, Johanna K Kaakinen, Suvi K Holm, and Aki Koponen. 2017. Digital game dynamics preferences and player types. Journal of Computer-Mediated Communication 22, 2 (2017), 88-103.

15. N Yee. 2015. Gamer motivation model overview and descriptions. Online: http://quanticfoundry. com/2015/12/15/handy-reference (2015). 